\title{
Pseudo-Valuations on UP-Algebras
}

\author{
Daniel A. Romano ${ }^{1}$
}

${ }^{1}$ International Mathematical Virtual Institute, Banja Luka, Bosnia and Herzegovina

\section{Article Info}

Keywords: UP-algebra, UP-ideal, proper UP-filter, pseudo-valuations 2010 AMS: 03G25, $03 C 05$

Received: 19 April, 2019

Accepted: 6 September 2019

Available online: 30 September 2019

\begin{abstract}
Looking at pseudo-valuations on some classes of abstract algebras, such as BCK, BCI, BCC and $\mathrm{KU}$, in this article we introduce the concept of pseudo-valuations on UP-algebras and analyze the relationship of these mappings with UP-substructures.
\end{abstract}

\section{Introduction}

The idea that universal algebra should be analyzed by means of pseudo-valuation was first developed by D. Busneag in 1996 [1]. This author has expanded the perception of pseudo-valuation on Hilbert's algebras [2]. Logical algebras and pseudo-valuations on them have become an object of interest for researchers in recent years. For example, Doh and Kang [3, 4] introduced in the concept of pseudo-valuation on BCK/BCI - algebras. Ghorbani in 2010 [5] determined a congruence on BCI - algebras based on pseudo-valuation and describe the obtained factorial structure generated by this congruence. Song, Roh and Jun described pseudo-valuation on BCK/BCI - algebras [12] and Song, Bordbar and Jun have described the quotient structure on such algebras generated by pseudo-valuation [13]. Jun, Lee and Song analyzed in article [8] several types of quasi-valuation maps on BCK - algebra and their interactions. Also, Mehrshad and Kouhestani were interested in pseudo-valuations on BCK - algebra [10]. Jun, Ahn and Roh. in [7] described pseudo-valuation on the BCC - algebras. Koam, Haider and Ansari described in 2019 pseudo-valuations on KU algebras [9].

The concept of UP-algebras is introduced and analyzed by Iampan in 2017 [6] as a generalization of the concept of KU - algebras. In this note, we offer one way of determining of pseudo-evaluation on PU - algebras. Apart from showing the features of this pseudo-valuation on UP-algebras, we have demonstrated how to construct a pseudo-metric space by such mapping.

\section{Preliminaries}

Here we give the definition of UP-algebra and some of its substructures necessary for further work.

Definition 2.1 ([6]). An algebra $A=(A, \cdot, 0)$ of type $(2,0)$ is called a UP-algebra if it satisfies the following axioms:

$(U P-1)(\forall x, y, z \in A)((y \cdot z) \cdot((x \cdot y) \cdot(x \cdot z))=0)$,

$(U P-2)(\forall x \in A)(0 \cdot x=x)$,

$(U P-3)(\forall x \in A)(x \cdot 0=0)$, and

$(U P-4)(\forall x, y \in A)((x \cdot y=0 \wedge y \cdot x=0) \Longrightarrow x=y)$.

In $A$ we can define a binary relation ' $\leqslant$ ' by

$$
(\forall x, y \in A)(x \leqslant y \Longleftrightarrow x \cdot y=0) .
$$

Definition 2.2 ([6]). A non-empty subset $J$ of a UP-algebra A is called a UP-ideal of A if it satisfies the following conditions:

(1) $0 \in J$, and

(2) $(\forall x, y, z \in A)((x \cdot(y \cdot z) \in J \wedge y \in J) \Longrightarrow x \cdot z \in J)$.

Definition 2.3 ([11]). Let A be a UP-algebra. A subset $G$ of $A$ is called a proper UP-filter of $A$ if it satisfies the following properties:

(3) $\neg(0 \in G)$, and

(4) $(\forall x, y, z \in A)((\neg(x \cdot(y \cdot z) \in G) \wedge x \cdot z \in G) \Longrightarrow y \in G)$. 


\section{The concept of pseudo-valuations on UP-algebras}

In this section, we introduce the concept of pseudo-valuations on UP-algebras, describe the basics properties of such pseudo-valuation and construct a pseudo-metric space based on this mapping.

Definition 3.1. A real-valued function $v$ on a UP-algebra A is called a pseudo-valuation on A if it satisfies the following two conditions:

(1) $v(0)=0$, and

(2) $(\forall x, y, z \in A)(v(x \cdot z) \leqslant v(x \cdot(y \cdot z))+v(y))$.

A pseudo-valuation $v$ on a UP-algebra A satisfying the following condition:

(3) $(\forall x \in A)(v(x)=0 \Longrightarrow x=0)$

is called a valuation on $X$.

Theorem 3.2. Let $v$ be a pseudo-valuation on a UP-algebra A. Then the following are valid:

(4) $(\forall x, y \in A)(v(y) \leqslant v(x \cdot y)+v(x))$,

(5) $(\forall x, y \in A)(v(x \cdot y) \leqslant v(y))$,

Proof. If we put $x=0, y=x$ and $z=y$ in formula (2), we get

$$
v(y) \leqslant v(x \cdot y)+v(x) .
$$

Thus, formula (4) is valid.

If we put $z=y$ in formula (2), we have $v(x \cdot y) \leqslant v(x \cdot(y \cdot y))+v(y)$ from which follows $v(x \cdot y) \leqslant v(y)$ due to the assertion (1) of Proposition 1.7 in [6], (UP-3) and (1). So, (5) is proven.

Corollary 3.3. Let $v$ be a pseudo-valuation on a UP-algebra A. Then

(6) $(\forall x, y \in A)(x \leqslant y \Longrightarrow v(y) \leqslant v(x))$.

Proof. Let $x$ and $y$ be arbitrary elements of a UP-algebra $A$ such that $x \leqslant y$. Then $x \cdot y=0$ and $v(x \cdot y)=0$ by (1). From here follows $v(y) \leqslant v(x \cdot y)+v(x)$ according to (4). Thus $v(y) \leqslant v(x)$. Thus, any pseudo-valuation on a UP-algebra is an inversely monotone mapping.

Corollary 3.4. Let $v$ be a pseudo-valuation on a UP-algebra A. Then

(7) $(\forall x \in A)(0 \leqslant v(x))$.

Proof. Since $x \cdot 0=0$ according to (UP-3), i.e. as always $x \leqslant 0$ in UP-algebra $A$, we have $0=v(0) \leqslant v(x)$ according to Corollary 3.3 .

Corollary 3.5. Let $v$ be a pseudo-valuation on a UP-algebra A. Then

(8) $(\forall x, y \in A)(v(x \cdot y) \leqslant v(x)+v(y))$.

Proof. Let $x$ and $y$ be arbitrary elements of $A$. Thus $v(x \cdot y) \leqslant y(y)$ by (5). Thus $v(x \cdot y) \leqslant v(x)+v(y)$ by Corollary 3.4.

Theorem 3.6. Let $v$ be a pseudo-valuation on a UP-algebra A. Then the set $J_{v}=\{x \in A: v(x)=0\}$ is an UP-ideal of A and the set $G=\{x \in A: 0<v(x)\}$ is a proper UP-filter of $A$.

Proof. Since $v(0)=0$, follows $0 \in J_{v}$.

Let $x, y$ and $z$ be arbitrary elements of $A$ such that $x \cdot(y \cdot z) \in J_{v}$ and $y \in J_{v}$. Then $v(x \cdot(y \cdot z))=0$ and $v(y)=0$. By (2) we have

$$
v(x \cdot z) \leqslant v(x \cdot(y \cdot z))+v(y)=0+0=0 .
$$

Thus $v(x \cdot z)=0$ according to Corollary 3.4. Hence $x \cdot z \in J_{v}$. So, the set $J_{v}$ is a UP-ideal of UP-algebra $A$.

The set $G$ is a proper UP-filter of $A$ by Theorem 3.7 in [11].

Corollary 3.7. Let $v$ be a pseudo-valuation on a UP-algebra A. Then $v$ is a valuation on $A$ if and only if $J_{v}=\{0\}$.

Proof. The claim follows from the definition of the concept of valuations on a UP-algebra $A$.

Remark 3.8. The previous corollary suggested that a valuation on an UP-algebra A can be defined if $\{0\}$ is a UP-ideal at A.

Example 3.9. For any ideal $J$ of a UP-algebra A, define a map $v_{J}: A \longrightarrow \mathbb{R}$ by $(\forall x \in J)\left(v_{J}(x)=0\right)$ and $(\forall x \in A \backslash J)\left(v_{J}(x) \in \mathbb{R}^{+}\right)$. Then, $v_{J}$ is a pseudo-valuation of $A$.

Example 3.10. Let $A=\{0,1,2,3,4\}$ be given and an operations on it as in Example 2.2 in [6]. Then $(A, \cdot, 0)$ is a UP-algebra. It is easy to directly verified that $v: A \longrightarrow \mathbb{R}$, given with $v(0)=v(1)=v(2)=0, v(3)=v(4)=3$, is a pseudo-valuation on $A$.

Theorem 3.11. Let $f:\left(A, \cdot, 0_{A}\right) \longrightarrow\left(B, *, 0_{B}\right)$ be a homomorphism of UP-algebras. If $v$ is a pseudo-valuation on $B$, then the composition $v \circ f$ is a pseudo-valuation on $A$.

Proof. First, we have $(v \circ f)\left(0_{A}\right)=v\left(f\left(0_{A}\right)\right)=v\left(0_{B}\right)=0$.

For any $x, y, z \in A$, we get $(v \circ f)(x \cdot z)=v(f(x \cdot z))=v(f(x) * f(z)) \leqslant v(f(x) *(f(y) * f(z)))+v(f(y))=(v \circ f)(x \cdot(y \cdot z))+(v \circ f)(y)$. Hence, $v \circ f$ is a pseudo-valuation on $A$.

Lemma 3.12. Suppose that $A$ is a UP-algebra. Then every pseudo-valuation $v$ on A satisfies the following inequality:

(9) $(\forall x, y, z \in A)(v(x \cdot z) \leqslant v(x \cdot y)+v(y \cdot z))$. 
Proof. From (UP-1) follows $y \cdot z \leqslant(x \cdot y) \cdot(x \cdot z)$. Thus $v(y \cdot z) \geqslant v((x \cdot y) \cdot(x \cdot z))$ by (6) and $v(y \cdot z) \geqslant v(x \cdot z)-v(x \cdot y)$ by (4). Therefore, $v(x \cdot z) \leqslant v(x \cdot y)+v(y \cdot z)$.

Now, we define pseudo-metric on UP-algebras and show related results.

Theorem 3.13. Let $A$ be a UP-algebra and $v$ be a pseudo-valuation on $A$. Then the mapping $d_{v}: A \times A \ni(x, y) \longmapsto v(x \cdot y)+v(y \cdot x) \in \mathbb{R}$ is a pseudo-metric on A.

Proof. Clearly, $0 \leqslant d_{v}(x, y) ; d_{v}(x, x)=0$ and $d_{v}(x, y)=d_{v}(y, x)$ for any $x, y \in A$. For any $x, y, z \in A$ from Lemma 3.12, we get that $d_{v}(x, y)+d_{v}(y, z)=$

$$
\begin{aligned}
& (v(x \cdot y)+v(y \cdot x))+(v(y \cdot z)+v(z \cdot y))= \\
& (v(x \cdot y)+v(y \cdot z))+(v(z \cdot y)+v(y \cdot x)) \geqslant \\
& v(x \cdot z)+v(z \cdot x)=d_{v}(x \cdot z) .
\end{aligned}
$$

Hence $\left(A, d_{v}\right)$ is a pseudo-metric space.

\section{Conclusion}

The aim of this paper was to study the concept of pseudo-valuation and their induced pseudo-metrics on UP - algebras. This work can be the basis for further and deeper research of the properties of UP - algebras.

\section{Acknowledgments}

The author wishes to express their sincere thanks to the referees for the valuable suggestions that led to an improvement of this paper.

\section{References}

[1] D. Busneag. Hilbert algebras with valuations. Math. Japon., 44 (2)(1996), 285-289.

[2] D. Busneag. On extensions of pseudo-valuations on Hilbert algebras. Discrete Math., 263(1-3)(2003), 11-24.

[3] M. I. Doh and M. S. Kang. BCK/BCI-algebras with pseudo-valuation. Honam Math. J., 32(2)(2010), $217-226$.

[4] M. I. Doh and M. S. Kang. Commutative pseudo valuations on BCK-algebras. Int. J. Math. Math. Sci., Vol. 2011, Article ID 754047, 6 pages

[5] S. Ghorbani. Quotient BCI-algebras induced by pseudo-valuations. Iranian J. Math. Sc. Inform., 5(2010), 13-24.

[6] A. Iampan. A new branch of the universal algebra: UP-algebras. Journal of Algebra and Related Topics, 5(1)(2017), 35-54.

[7] Y. B. Jun, S. S. Ahn amd E. H. Roh. BCC-algebras with pseudo-valuations. Filomat, 26(2)(2012), $243--252$.

[8] Y. B. Jun, K. J. Lee and S. Z. Song. Quasi-valuation maps based on positive implicative ideals in BCK-algebras. Algebra and Discrete Mathematics, 26(1)(2018), 65--75.

[9] A. N.A. Koam, A. Haider and M. A. Ansari. Pseudo-metric on KU-algebras. Korean J. Math., 27(1)(2019), 131-140.

[10] S. Mehrshad and N. Kouhestani. On pseudo-valuations on BCK-algebras. Filomat, 32(12)(2018), 4319--4332.

[11] D. A. Romano. Proper UP-filters in UP-algebra. Universal Journal of Mathematics and Applications, 1(2)(2018), 98-100.

[12] S.-Z. Song, E. H. Roh and Y. B. Jun. Quasi-valuation maps on BCK/BCI-algebras. Kyungpook Math. J., 55(4)(2015), 859-870.

[13] S.-Z. Song, H. Bordbar and Y. B. Jun. Quotient structures of BCK/BCI-algebras induced by quasi-valuation maps. Axioms 2018, 7(2): 26. 11pages 